\title{
Performances des butées hydrodynamiques à géométrie fixe : influence des effets thermiques et des déformations mécaniques
}

\author{
Said Abdallah Ahmed ${ }^{\mathrm{a}}$ et Michel Fillon \\ Université de Poitiers, Laboratoire de Mécanique des Solides, UMR CNRS 6610, SP2MI, Boulevard Marie et Pierre Curie, \\ Téléport 2, BP 30179, 86962 Futuroscope Chasseneuil Cedex, France
}

Reçu le 15 janvier 2007, accepté le 23 mai 2007

\begin{abstract}
Résumé - L'influence des effets thermiques et des déformations mécaniques sur les performances des butées hydrodynamiques à géométrie fixe est analysée. Les études menées sur les butées hydrodynamiques à géométrie fixe allient rarement les effets thermiques et les déformations. Après un bref rappel théorique de la lubrification thermohydrodynamique (THD), nous décrivons les méthodes de calculs des déformations mécaniques. L'étude thermoélastohydrodynamique (TEHD), qui prend en compte les effets thermiques locaux et les déformations mécaniques, est réalisée sur une butée hydrodynamique à géométrie fixe à huit patins. Les champs de pression hydrodynamique et de température sont obtenus par une méthode des différences finies et le champ des déplacements de la surface des patins est obtenu à l'aide de la méthode des éléments-finis. Les déformations mécaniques du grain mobile sont également prises en compte pour résoudre le problème TEHD. L'influence des conditions de fonctionnement (charge appliquée et vitesse de rotation) sur les différentes caractéristiques de la butée est également analysée. Il ressort que pour déterminer précisément les caractéristiques de fonctionnement d'une butée hydrodynamique à patins fixes, il ne faut pas négliger les déformations mécaniques du collet et en particulier, lorsque la charge appliquée est élevée.
\end{abstract}

Mots clés : Tribologie / lubrification hydrodynamique / butée à patins fixes / effets thermiques / déformations mécaniques

\begin{abstract}
Performance of hydrodynamic fixed geometry thrust bearings: influence of thermal effects and mechanical deformations. The influence of thermal effects and mechanical deformations on the performance of hydrodynamic fixed geometry thrust bearings is analyzed. Previous studies on fixed geometry hydrodynamic thrust bearings rarely combined thermal effects and deformations. After a short presentation of the theory of thermohydrodynamic (THD) lubrication, we will describe the methods of mechanical deformation calculations. The thermoelastohydrodynamic (TEHD) study, which takes into account the local thermal effects and the mechanical deformations, is carried out on an eight pad fixed geometry hydrodynamic thrust bearing. The hydrodynamic pressure and temperature fields are obtained by the mean of finite difference method and the pad displacement field is computed using the finite element method. We also take into account the mechanical deformations of the runner in order to solve the TEHD problem. The influence of the operating conditions (applied load and rotation speed) on the various characteristics of the thrust bearing is also analyzed. We showed that mechanical deformations of the runner were found very significant for an accurate prediction of the performance of the thrust bearing.
\end{abstract}

Key words: Tribology / hydrodynamic lubrication / fixed pad thrust bearings / thermal effects / mechanical deformations

\section{Introduction}

Les études des phénomènes thermiques dans les butées hydrodynamiques à géométrie fixe sont peu nombreuses. L'augmentation de la température a cependant

\footnotetext{
a Auteur correspondant : ahmed@lms.univ-poitiers.fr
}

une grande influence sur les caractéristiques des paliers et des butées. Pour une butée très chargée, ce sont l'épaisseur minimale du film et la pression maximale qui sont les paramètres critiques. Une pression maximale très élevée implique des déplacements élastiques significatifs. Dans des conditions de fonctionnement sévères, 


\section{Nomenclature}

\begin{tabular}{|c|c|c|}
\hline$C_{\mathrm{p}}$ & chaleur spécifique du lubrifiant & $\mathrm{J} . \mathrm{kg}^{-1} \cdot \mathrm{K}^{-1}$ \\
\hline$e_{\mathrm{p}}$ & épaisseur du patin & $\mathrm{m}$ \\
\hline$h$ & épaisseur locale du film lubrifiant & $\mathrm{m}$ \\
\hline$h_{\text {huile }}$ & coefficient global d'échange thermique & $\mathrm{W} \cdot \mathrm{m}^{-2} \cdot \mathrm{K}^{-1}$ \\
\hline$h_{\mathrm{e}}$ & épaisseur du film à l'entrée du patin & $\mathrm{m}$ \\
\hline$h_{\mathrm{s}}$ & épaisseur du film à la sortie du patin & $\mathrm{m}$ \\
\hline$D_{\mathrm{i}}$ & largeur de la partie inclinée & $\mathrm{m}$ \\
\hline$K_{\mathrm{h}}$ & conductivité thermique du lubrifiant & $\mathrm{W} \cdot \mathrm{m}^{-1} \cdot \mathrm{K}^{-1}$ \\
\hline$n$ & nombre de patins de la butée & \\
\hline$N$ & vitesse de rotation du collet & $\operatorname{tr} . \min ^{-1}$ \\
\hline$P$ & pression hydrodynamique dans le film & $\mathrm{Pa}$ \\
\hline$Q_{\mathrm{e}}$ & débit tangentiel à l'entrée du patin & $\mathrm{m}^{3} \cdot \mathrm{s}^{-1}$ \\
\hline$Q_{\operatorname{Re}}$ & débit radial au rayon extérieur du patin & $\mathrm{m}^{3} \cdot \mathrm{s}^{-1}$ \\
\hline$Q_{\mathrm{Ri}}$ & débit radial au rayon intérieur du patin & $\mathrm{m}^{3} \cdot \mathrm{s}^{-1}$ \\
\hline$Q_{\mathrm{s}}$ & débit tangentiel à la sortie du patin & $\mathrm{m}^{3} \cdot \mathrm{s}^{-1}$ \\
\hline$(r, \theta, z)$ & coordonnées & $(\mathrm{m}, \mathrm{rad}, \mathrm{m})$ \\
\hline$R_{\mathrm{e}}$ & rayon externe de la butée & $\mathrm{m}$ \\
\hline$R_{\mathrm{i}}$ & rayon interne de la butée & $\mathrm{m}$ \\
\hline$T$ & température dans le film & ${ }^{\circ} \mathrm{C}$ \\
\hline$T_{0}$ & température d'alimentation du lubrifiant & ${ }^{\circ} \mathrm{C}$ \\
\hline$T_{\mathrm{p}}$ & température dans le patin & ${ }^{\circ} \mathrm{C}$ \\
\hline$(u, v, w)$ & composantes du vecteur vitesse dans le film & $\mathrm{m} \cdot \mathrm{s}^{-1}$ \\
\hline$W$ & module de la charge appliquée & $\mathrm{N}$ \\
\hline$\alpha$ & angle d'inclinaison du plan incliné & $\mathrm{rad}$ \\
\hline$\beta$ & coefficient de thermoviscosité & $\mathrm{K}^{-1}$ \\
\hline$\mu$ & viscosité dynamique du lubrifiant & Pa.s \\
\hline$\theta_{\mathrm{e}}$ & coordonnée angulaire de l'entrée du patin & $\mathrm{rad}$ \\
\hline$\theta_{\mathrm{s}}$ & coordonnée angulaire de la sortie du patin & $\mathrm{rad}$ \\
\hline$\rho$ & masse volumique du lubrifiant & $\mathrm{kg} \cdot \mathrm{m}^{-3}$ \\
\hline$\delta_{\mathrm{c}}$ & déplacement axial du collet & $\mathrm{m}$ \\
\hline$\delta_{\mathrm{p}}$ & déplacement axial du patin & $\mathrm{m}$ \\
\hline$\omega$ & vitesse angulaire du collet & $\operatorname{rad} . \mathrm{s}^{-1}$ \\
\hline
\end{tabular}

l'augmentation de la température peut modifier les performances de la butée. Ainsi, une baisse de la capacité de charge due à la diminution de la viscosité du lubrifiant peut être observée. La déformation mécanique des surfaces du contact influe sur la géométrie du film et donc sur les performances. Nous ne pouvons pas ignorer les déformations mécaniques dues au champ de pression hydrodynamique, ni utiliser une température constante pour décrire le comportement de la butée; une analyse thermohydrodynamique (THD) est donc nécessaire pour prendre en compte la variation locale de la viscosité avec la température. Le régime THD est caractérisé par la résolution simultanée de l'équation de Reynolds généralisée et l'équation de l'énergie en tenant compte de tous les transferts de chaleur dans le mécanisme. Ce problème a été résolu par différentes méthodes, principalement pour les paliers hydrodynamiques. La plus précise est l'analyse thermoélastohydrodynamique qui permet de résoudre simultanément l'équation de Reynolds généralisée et l'équation de l'énergie, en tenant compte des transferts de chaleur dans les solides, des conditions d'alimentation à l'entrée des contacts (recirculation du lubrifiant) et de la déformation locale des solides.

Pinkus [1], Khonsari [2], Fillon [3] et plus récemment Tanaka [4] présentent une bibliographie retraçant l'évolution des études THD sur le plan théorique et expérimental. Le couplage de l'équation de Reynolds et de l'énergie dans le film ajoute une difficulté supplémentaire dans la résolution des problèmes THD. En effet, la pression dans le film d'huile dépend de la viscosité qui est elle-même fonction de la température.

Dadouche et Fillon [5,6] ont effectué la première analyse expérimentale des effets thermiques dans une butée hydrodynamique à géométrie fixe. Cette butée comportait 8 patins composés d'un plan incliné et d'un plan parallèle. Le dispositif expérimental conçu pour cette étude, a permis d'étudier l'influence d'une charge imposée, de la vitesse de rotation et de la température d'alimentation sur les champs de pression et de température, les débits et l'épaisseur minimale du film lubrifiant. Les résultats expérimentaux ont été confrontés aux résultats thermohydrodynamiques issus de simulations numériques. La concordance était globalement satisfaisante et les différences observées ont été attribuées principalement à la non prise en compte des déformations thermiques et mécaniques dans le modèle théorique.

Ettles [7] a étudié les effets thermoélastiques (transitoires) sur une butée composée de patins oscillants de longueur infinie. L'équation de l'énergie est résolue à l'aide de la méthode des volumes finis en tenant compte de 
l'écoulement inverse. Toutefois, les hypothèses faites sont très élémentaires. En effet, la théorie des poutres est utilisée pour déterminer les déformations TEHD. Dans un autre article, Ettles et Advani [8] ont montré que la prise en compte des déformations thermiques des patins oscillants se traduisait par une réduction significative de la capacité de charge.

Une analyse TEHD tenant compte de la déformation du collet a été menée par Brockett et al. [9]. Dans cette étude, une comparaison entre trois modèles (adiabatique, THD et TEHD) est présentée. Pour la solution adiabatique, et dans le cas d'un collet d'épaisseur de $12,7 \mathrm{~mm}$, la prise en compte des déformations du collet change sensiblement les caractéristiques de la butée telle que l'épaisseur minimale du film qui chute et la température maximale qui augmente. Par contre, pour un collet d'épaisseur 25,4 mm, l'influence des déformations est négligeable. Pour l'analyse THD, l'épaisseur minimale $\mathrm{du}$ film est plus importante à fortes charges par rapport à celle calculée avec une condition adiabatique. Cela est dû aux transferts thermiques par conduction qui permet de baisser la température dans le film lubrifiant. Même avec des chargements plus importants, les déformations mécaniques sont relativement moins importantes par rapport aux déformations thermiques. Pour le cas de charge de $22,4 \mathrm{kN}$, les déplacements d'origine mécanique ont conduit à un écart maximal de $8 \%$ sur l'épaisseur minimale du film par rapport au régime adiabatique tandis que les déplacements thermiques ont engendré un écart maximal de $45 \%$ de l'épaisseur minimale du film.

Huebner [10] a réalisé une analyse numérique THD d'une butée à géométrie fixe. Il a pris en compte la variation de la viscosité avec la température suivant les trois directions. Il a observé que lors du passage du régime laminaire au régime turbulent, la température baisse sensiblement. Dans une autre étude THD [11], toujours dans la même année, il a ensuite montré que les performances de la butée sont dominées par le type du lubrifiant, la température d'alimentation, la vitesse de rotation et le rapport de l'épaisseur du film à l'entrée à celle à la sortie. Par ailleurs, il a noté que le fait d'imposer une condition de type adiabatique à l'interface film/patin, permet de prédire précisément les performances des butées.

Récemment Dadouche et al. [12] ont effectué une étude THD d'une butée à géométrie fixe. Ils ont procédé à une comparaison entre les résultats numériques et expérimentaux. L'influence de la température du film d'huile, de la charge appliquée et de l'épaisseur minimale du film sur les performances d'une butée est analysée et discutée. La confrontation des résultats expérimentaux aux résultats numériques est globalement satisfaisante. Ils ont montré, qu'en général, environ $80 \%$ à $90 \%$ de la chaleur produite par frottement est évacuée de la butée par le débit d'huile, la chaleur restante étant transmise par conduction à travers les solides.

Dans cette étude, nous évaluons l'influence des effets thermiques locaux et des déformations mécaniques sur les performances des butées hydrodynamiques à géométrie fixe. L'étude est réalisée sur la butée à huit

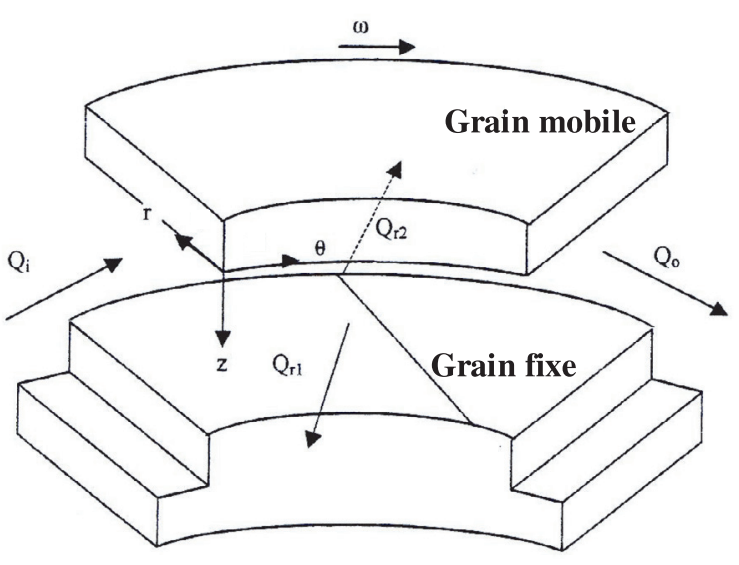

Fig. 1. Système de coordonnées.

patins testée par Dadouche [5]. Aussi, après un bref rappel théorique de la lubrification thermohydrodynamique (THD), nous décrivons les méthodes de calculs des déformations mécaniques. Les champs de pression hydrodynamique et de température sont obtenus par une méthode des différences finies et le champ des déplacements de la surface des patins est obtenu à l'aide de la méthode des éléments-finis. Les déformations mécaniques du grain mobile sont prises en compte pour résoudre le problème TEHD. L'influence des conditions de fonctionnement (charge appliquée et vitesse de rotation) sur les différentes caractéristiques de la butée est également analysée.

\section{2 Étude théorique}

Les équations de base des problèmes thermohydrodynamique (THD) et thermoélastohydrodynamique (TEHD) sont écrites en coordonnées cylindriques. Les conditions aux limites associées sont également précisées.

\subsection{Analyse THD}

\subsection{1 Équation de Reynolds généralisée}

Dans le repère lié à la butée (Fig. 1), l'équation de Reynolds généralisée pour un fluide newtonien et en supposant un régime d'écoulement laminaire et stationnaire s'écrit :

$$
\frac{\partial}{\partial r}\left(G_{1} r \frac{\partial p}{\partial r}\right)+\frac{1}{r} \frac{\partial}{\partial \theta}\left(G_{1} \frac{\partial p}{\partial \theta}\right)=r \omega \frac{\partial G_{2}}{\partial \theta}
$$

Le plan $(O, r, \theta)$ est confondu avec la surface active du grain mobile animé d'une vitesse de rotation $\omega$ autour de l'axe $z$ du repère. Les fonctions $G_{1}$ et $G_{2}$ sont définies par :

$$
\begin{gathered}
G_{1}=\int_{0}^{h} \frac{h}{\mu}\left[z-\frac{I_{2}}{J_{2}}\right] \mathrm{d} z \text { et } G_{2}=\frac{1}{J_{2}} \int_{0}^{h} \frac{z}{\mu} \mathrm{d} z \\
\text { avec }: I_{2}=\int_{0}^{h} \frac{z}{\mu} \mathrm{d} z \text { et } J_{2}=\int_{0}^{h} \frac{1}{\mu} \mathrm{d} z
\end{gathered}
$$




\subsection{2 Équation de l'énergie}

L'équation de l'énergie permet de calculer le champ de température dans le film fluide. Elle traduit la conservation d'énergie et permet d'étudier localement les transferts thermiques existant dans la zone qui sépare le collet et le grain fixe.

L'équation de l'énergie s'écrit :

$$
\begin{aligned}
\underbrace{\rho C_{\mathrm{p}}\left[u \frac{\partial T}{\partial r}+\frac{v}{r} \frac{\partial T}{\partial \theta}+w \frac{\partial T}{\partial z}\right]}_{\text {Convection }} & = \\
\underbrace{\mu\left[\left(\frac{\partial u}{\partial z}\right)^{2}+\left(\frac{\partial v}{\partial z}\right)^{2}\right]}_{\text {Dissipation visqueuse }} & +\underbrace{K_{\mathrm{h}} \frac{\partial^{2} T}{\partial z^{2}}}_{\text {Conduction }}
\end{aligned}
$$

où $T(r, \theta, z)$ est la température en un point quelconque du film et chaque terme caractérise un des principaux types de transferts thermiques qui ont lieu dans le film lubrifiant.

L'écoulement d'un film mince visqueux ainsi décrit est stationnaire de masse volumique $\rho$ constante, de coefficient de conductivité $K_{\mathrm{h}}$ constant et de coefficient de dilatation thermique $\alpha$ nul. L'hypothèse d'épaisseur de film très mince permet de négliger les termes de conduction radiale et tangentielle dans l'équation de l'énergie et seul le terme de conduction axiale reste prépondérant.

\subsection{3 Équation de la chaleur}

Afin de tenir compte des transferts thermiques dans les patins (ou grain fixe) par conduction, il est nécessaire de résoudre l'équation de la chaleur.

En régime permanent et sans source interne de chaleur, nous obtenons l'équation de la chaleur qui se réduit à l'équation appelée « équation de Laplace » que nous écrivons dans le repère $(O, r, \theta, z)$ associé au patin :

$$
\frac{1}{r} \frac{\partial T_{\mathrm{p}}}{\partial r}+\frac{\partial^{2} T_{\mathrm{p}}}{\partial r^{2}}+\frac{1}{r^{2}} \frac{\partial^{2} T_{\mathrm{p}}}{\partial \theta^{2}}+\frac{\partial^{2} T_{\mathrm{p}}}{\partial z^{2}}=0
$$

\subsubsection{Variation de la viscosité avec la température}

La viscosité des lubrifiants diminue très rapidement quand la température augmente. La connaissance de cette variation est nécessaire pour le praticien. On peut utiliser une relation exponentielle liant la viscosité à la température, soit :

$$
\mu(T)=\mu_{0} \mathrm{e}^{-\beta\left[T-T_{0}\right]}
$$

où $\mu_{0}$ est la viscosité dynamique à une température donnée, $T$ la température absolue et $\beta$ propre à chaque lubrifiant, appelé, coefficient de thermoviscosité et peut être déterminé à partir de la viscosité connue à deux températures différentes.

\subsubsection{Conditions aux limites}

\subsubsection{Pression}

On suppose que la pression de référence est la pression atmosphérique. La pression d'entrée et de sortie du film ainsi que celle aux rayons intérieur et extérieur de la butée sont égales à la pression de référence, l'alimentation étant réalisée par barbotage.

\subsubsection{Température}

Les transferts thermiques sont une combinaison des différents modes de transmission de chaleur qui interviennent dans la butée : la convection, la conduction et le rayonnement. Ceux-ci peuvent intervenir partout dans le système. La température d'entrée est déterminée à partir d'un bilan des flux de chaleur dans la rainure qui tient compte du mélange du fluide frais qui est apporté par l'alimentation et de la circulation d'huile chaude provenant du patin précédent.

À l'interface film/patin, les dimensions du film étant très faibles devant celles du patin, on considère que l'échange ne se fait que par conduction. On applique donc une continuité des flux de chaleur et on considère que les températures du film et du patin sont les mêmes au niveau de l'interface.

À l'extérieur de la butée, l'échange s'effectue par convection avec l'huile mais aussi par rayonnement : ceci est caractérisé par un coefficient global d'échange thermique.

\subsection{Analyse TEHD}

La déformation mécanique des surfaces du contact influe sur la géométrie du film et donc sur les performances de la butée. L'approche la plus précise est donc l'analyse TEHD qui permet de réaliser une étude THD en tenant compte des déformations mécaniques locales des solides.

\subsubsection{Théorie de l'élasticité}

Les déformations auxquelles nous allons nous intéresser sont des déformations mécaniques qui interviennent au cours du fonctionnement de la butée. Ces déformations élastiques engendrées par la pression hydrodynamique modifient la forme géométrique de la butée en induisant une modification de l'épaisseur du film. Le code que nous utilisons pour le calcul des déformations est CASTEM2000, qui intègre les équations classiques de l'élasticité :

$$
\overline{\bar{\sigma}}=\frac{E}{1+\nu}\left(\overline{\bar{\varepsilon}}+\frac{\nu}{1-2 \nu} \operatorname{Tr}(\overline{\bar{\varepsilon}}) G\right)
$$

L'équation ci-dessus nous donne l'expression du tenseur des contraintes en fonction des déformations, c'est la 
loi de Hooke généralisée. Ainsi, nous déduisons l'expression du tenseur des déformations en fonction de celle des contraintes, soit :

$$
\overline{\bar{\varepsilon}}=\frac{1+\nu}{E}-\frac{\nu}{E} \operatorname{Tr}(\overline{\bar{\sigma}}) G
$$

avec :

$$
G=\frac{E}{2(1+\nu)}
$$

$\overline{\bar{\varepsilon}}$ : Tenseur des déformations

$\overline{\bar{\sigma}}$ : Tenseur des contraintes

$E$ : Module d'Young

$\nu$ : Coefficient de Poisson.

Le tenseur des contraintes étant connu, la loi de comportement élastique isotrope nous a permis de calculer le tenseur des petites déformations $\overline{\bar{\varepsilon}}$. Il existe donc un champ de déplacement $\delta$ tel que :

$$
\overline{\bar{\varepsilon}}=\frac{1}{2}\left[\operatorname{grad} \overline{\bar{\delta}}+\operatorname{grad}^{t} \overline{\bar{\delta}}\right]
$$

car les équations de Beltrami garantissent l'intégrabilité de $\overline{\bar{\varepsilon}}$. À l'aide de la mécanique des milieux continus, on calcule d'abord le tenseur des rotations antisymétrique, puis les champs des déplacements axiaux des surfaces du collet $\delta_{\mathrm{c}}(r, \theta)$ et du patin $\delta_{\mathrm{p}}(r, \theta)$. Ces déplacements sont utilisés pour définir l'épaisseur du film lubrifiant en régime TEHD dans les équations (8) et (9).

\subsubsection{Conditions aux limites sur les déplacements}

Les équations que nous venons de présenter nous permettent de décrire le comportement de la butée soumis à diverses conditions de fonctionnement. Pour les résoudre, il est nécessaire d'appliquer des conditions aux limites adéquates.

En ce qui concerne le grain fixe, nous avons utilisé les conditions du banc d'essai (Figs. 2a et b) : la face inférieure du grain fixe est supposée en appui simple sur le disque support (déplacement axial positif ou nul) avec une prise en compte des vis de fixation. Le champ de pression hydrodynamique est appliqué sur la surface des patins (Fig. 2a).

De plus, on considère que les nœuds de cette face appartenant aux vis sont totalement bloqués (condition d'encastrement de la section des deux vis). Enfin, des conditions de symétrie sont utilisées pour prendre en compte la continuité de la butée : on suppose que les bords circonférentiels de la demi-butée sont de telle manière qu'ils ne peuvent effectuer de rotation $(\delta \theta=0)$. La prise en compte des vis de fixation du grain fixe nous a permis de montrer l'influence de celles-ci sur les déplacements axiaux des patins.

Concernant le grain mobile, les conditions utilisées consistent en un encastrement dans une structure rigide au rayon intérieur de la butée et le bord extérieur du grain mobile reste libre de se déformer.

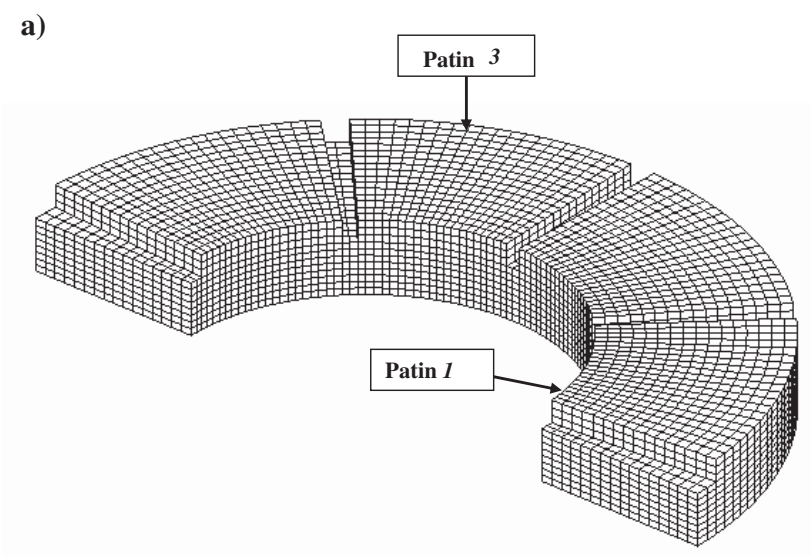

b)

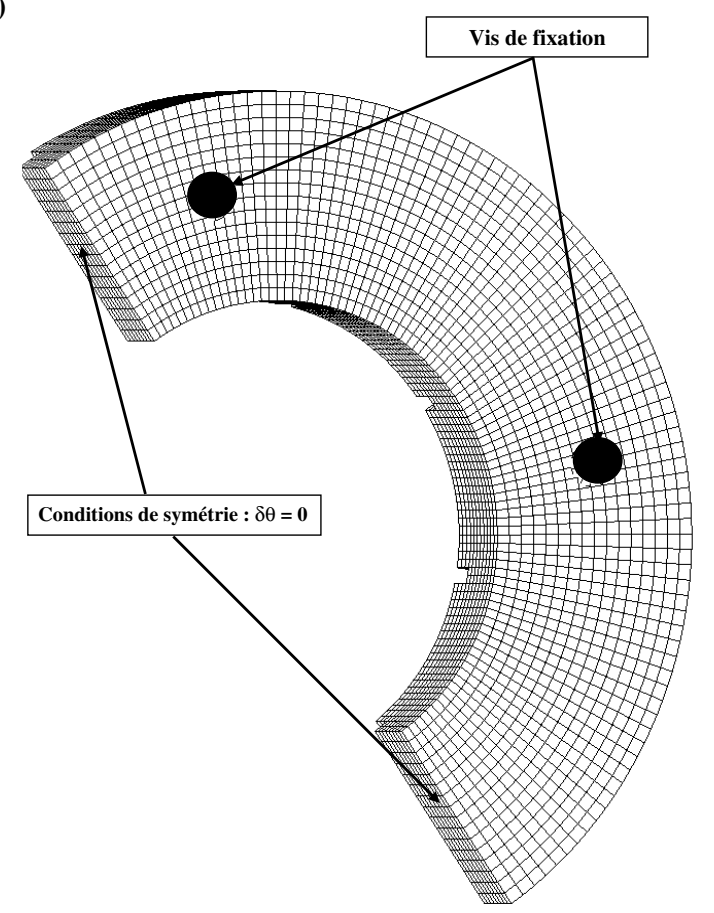

Fig. 2. a) Modélisation d'une demi-butée : géométrie des patins. b) Modélisation d'une demi-butée avec vis de fixation.

\subsection{3 Épaisseur du film lubrifiant}

Chaque patin comprend une partie inclinée et une partie parallèle. La première partie ayant une pente très faible permet la formation d'un film lubrifiant dont la pression hydrodynamique est générée par la rotation du collet.

Pour une butée alignée, l'épaisseur du film d'huile (Fig. 3) s'écrit :

- Partie du plan incliné :

$$
h=h_{\mathrm{e}}-\frac{h_{\mathrm{e}}-h_{\mathrm{s}}}{D_{\mathrm{i}}} r \sin \theta+\delta_{\mathrm{c}}(r, \theta)+\delta_{\mathrm{p}}(r, \theta)
$$

- Partie du plan parallèle :

$$
h=h_{\mathrm{s}}+\delta_{\mathrm{c}}(r, \theta)+\delta_{\mathrm{p}}(r, \theta)
$$



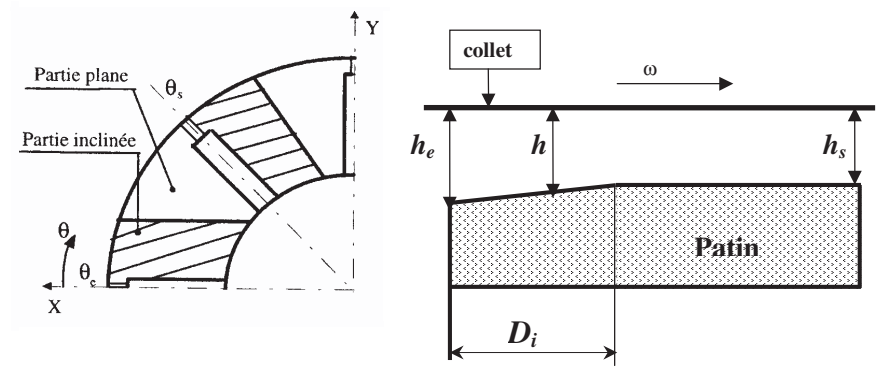

Fig. 3. Schéma d'un patin et épaisseur du film.

\subsection{Résolution numérique}

Le comportement TEHD d'une butée hydrodynamique à géométrie fixe est déterminé par la résolution de l'ensemble des équations déjà présentées auxquelles il convient d'ajouter les conditions aux limites associées.

\subsubsection{Méthode numérique de résolution}

L'équation de Reynolds généralisée (1) est une équation aux dérivées partielles elliptiques dont l'inconnue principale est la pression : les différences finies centrées sont utilisées et la résolution est effectuée par la méthode de Gauss-Seidel avec coefficient de surrelaxation.

L'équation de l'énergie (2) est une équation aux dérivées partielles paraboliques à valeur initiale dans laquelle la coordonnée angulaire joue le rôle du temps dans les problèmes non stationnaires. Une méthode de différences finies avec schéma implicite a été utilisée et la résolution est effectuée à l'aide d'un algorithme mis au point par Richtmyer [13].

L'équation de la chaleur (3) est une équation aux dérivées partielles elliptiques, sa résolution est effectuée par la méthode de Gauss-Seidel.

Les déplacements des surfaces des patins et du collet sont déterminés numériquement par la méthode des éléments-finis, à l'aide du logiciel CASTEM2000.

\subsubsection{Schéma de résolution du programme de calcul TEHD}

L'organigramme des calculs TEHD est présenté sur la figure 4. Dans la simulation numérique par élémentsfinis, pour résoudre le problème TEHD, des éléments hexaédriques à 8 nœuds ont été utilisés. Ils sont suffisants pour une bonne précision des calculs, avec un temps moins long par rapport aux éléments à 20 nœuds. Nous avons utilisé un maillage comportant 90 éléments circonférentiels au sein de la demi-butée, 20 éléments circonférentiels au niveau du patin, 16 éléments radiaux, 11 selon l'épaisseur des patins. Un exemple de maillage du grain fixe est présenté à la figure $2 \mathrm{a}$.

Avant de procéder aux calculs numériques des différentes butées testées, nous avons procédé à une série de calculs de validation.

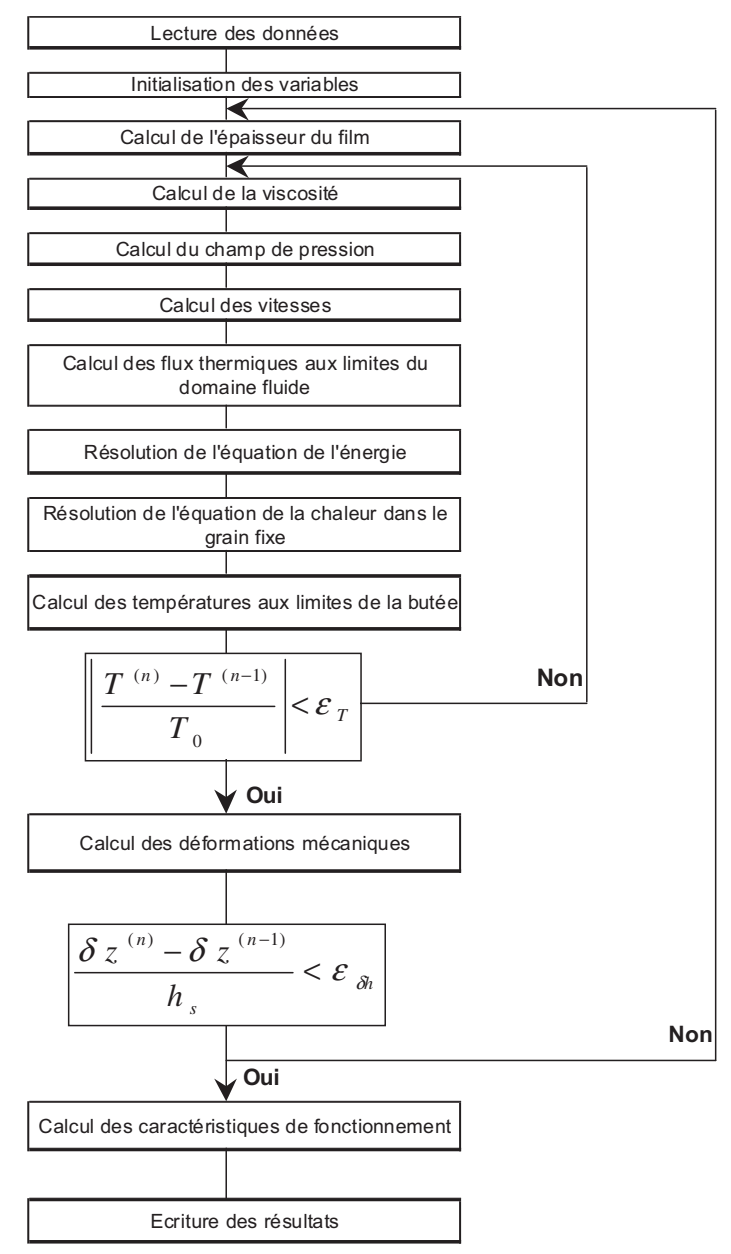

Fig. 4. Organigramme général du calcul TEHD.

La méthode utilisée pour analyser l'influence du maillage sur les résultats numériques, consiste à affiner le maillage par étapes successives, dans le but d'obtenir une faible variation de la solution pour une importante augmentation du nombre d'éléments du maillage. La solution est représentée par une valeur caractéristique, qui est, dans notre étude, le déplacement axial. En représentant l'évolution de cette valeur caractéristique en fonction du nombre d'éléments du maillage, le calcul est jugé correct lorsque cette valeur atteint une asymptote. Nous avons procédé à des maillages suivant les différentes directions (radiale et angulaire) pour un champ de pression équivalent d'une charge de $8 \mathrm{kN}$ et une vitesse de rotation de 2600 tr. $\min ^{-1}$.

Ensuite, le calcul des déplacements a été validé par la théorie classique de la résistance des matériaux sur des poutres en compression.

\section{Résultats}

\subsection{Caractéristiques générales de la butée}

Les principales caractéristiques géométriques du grain fixe sont présentées dans le tableau 1. 
Tableau 1. Caractéristiques géométriques de la butée.

\begin{tabular}{llc}
\hline Nombre de patins & $n_{\mathrm{p}}$ & 8 \\
\hline Amplitude angulaire du patin & $C_{\mathrm{patin}}$ & $38^{\circ}$ \\
\hline Rayon extérieur du grain fixe & $R_{\text {ext }}$ & $0,100 \mathrm{~m}$ \\
\hline Rayon intérieur du grain fixe & $R_{\text {int }}$ & $0,053 \mathrm{~m}$ \\
\hline Largeur de la partie inclinée & $D_{\mathrm{i}}$ & $0,027 \mathrm{~m}$ \\
\hline Hauteur de la partie inclinée & $h_{\mathrm{e}}-h_{\mathrm{s}}$ & $95 \mu \mathrm{m}$ \\
\hline Épaisseur du grain fixe & $E_{\mathrm{p}}$ & $0,020 \mathrm{~m}$ \\
\hline Épaisseur du collet & $E_{\mathrm{c}}$ & $0,015 \mathrm{~m}$ \\
\hline Rayon extérieur du collet & $R_{\mathrm{c}}$ & $0,120 \mathrm{~m}$ \\
\hline
\end{tabular}

Le grain fixe est en bronze (CuSn8P) et le grain mobile est en acier. Les caractéristiques des matériaux et du lubrifiant ainsi que les conditions de fonctionnement sont présentées dans les tableaux 2 à 4 .

\subsection{Influence des déformations mécaniques}

Afin d'analyser l'influence de la prise en compte des déformations mécaniques (induites par le champ de pression hydrodynamique) sur les résultats numériques, les évolutions des déplacements axiaux au centre du patin suivant les directions radiales et circonférentielles sont présentées et analysées. Une comparaison entre les modèles TEHD (qui tiennent compte des déformations mécaniques) et THD, pour diverses conditions de fonctionnement, est également effectuée.

\subsubsection{Déformations mécaniques du grain fixe}

Le code de calcul par éléments-finis que nous utilisons nous permet d'obtenir le champ de déplacements. Seuls les déplacements axiaux des nœuds de la surface du grain fixe sont utilisés pour définir la géométrie du film en fonctionnement.

La figure 5 illustre ces déplacements à la surface du grain fixe sur 4 patins. Les résultats que nous présentons dans la suite sont tous issus des déplacements de cette zone.

Les évolutions des déplacements axiaux, au rayon moyen et à mi-amplitude angulaire du patin sont données par les courbes des figures 6 et 7 . Ces déplacements sont présentés en fonction des directions radiale et circonférentielle, pour différentes charges appliquées sur la butée.

\subsubsection{Déformations mécaniques du collet}

En utilisant la même démarche que celle utilisée pour le grain fixe, la répartition radiale des déplacements axiaux de la surface du collet dans la section correspondant à la mi-amplitude du patin est analysée pour différentes charges appliquées. Les résultats correspondants sont présentés à la figure 8 .
Les figures 7 et 8 montrent que ce sont principalement les déplacements du grain mobile qui vont fortement influencer l'épaisseur du film lubrifiant et donc les performances de la butée.

\subsubsection{Influence des déformations mécaniques sur le champ de pression}

La pression hydrodynamique a été mesurée par Dadouche $[6]$ en 5 points différents. Le point $P_{2}(r=$ $77,5 \mathrm{~mm}, \theta=19^{\circ}$ ) représente le point où le champ de pression prend sa valeur maximale mesurée. La figure 9 représente la variation de la pression, en ce point, avec la charge appliquée pour une vitesse de rotation constante de 2600 tr. $\mathrm{min}^{-1}$. Une comparaison entre le champ de pression hydrodynamique prédit à l'aide des théories THD et TEHD, avec prise en compte des déformations mécaniques, et les mesures effectuées localement au point $P_{2}$ a été réalisée. En imposant une vitesse de rotation constante à 2600 tr.min ${ }^{-1}$ (Fig. 9), la correspondance entre les résultats TEHD et les expériences réalisées est bonne. Pour des charges allant de 1 à $8 \mathrm{kN}$, les écarts obtenus entre le modèle TEHD et les expériences effectuées dans le dispositif expérimental, limité à $8 \mathrm{kN}$, varient entre $8 \%$ et $20 \%$. Les résultats issus des modèles numériques THD et TEHD sont proches et leurs écarts n'excèdent pas $2 \%$. Par ailleurs, les valeurs de la pression calculée au point $P_{2}$ par les modèles TEHD et THD augmentent avec la charge appliquée. Les valeurs issues de l'approche TEHD restent inférieures à celles prédites par les calculs THD; l'écart augmente avec la charge mais l'allure générale des courbes reste inchangée. Pour les charges élevées, la courbe TEHD est plus proche de la courbe issue des expériences (extrapolation pour les charges supérieures à $8 \mathrm{kN}$ ). Les déformations mécaniques entraînent une diminution de la pression maximale de $2 \%$ par rapport au modèle THD pour une charge appliquée de $25 \mathrm{kN}$.

Pour une vitesse de rotation élevée de 2600 tr.min ${ }^{-1}$, l'évolution de la répartition du champ de pression, suivant la direction radiale, calculée sur la surface du patin à mi-amplitude angulaire est donnée par les courbes de la figure 10. On constate que les écarts entre la théorie THD et la théorie TEHD sont sensibles pour les fortes charges. On ne peut donc pas négliger les déformations mécaniques pour les charges élevées.

Les courbes présentées dans la figure 11 montrent les champs de pression prédits à l'aide de la théorie thermoélastohydrodynamique (TEHD) avec une prise en compte des déformations mécaniques pour différentes conditions de fonctionnement (charges et vitesses de rotation). En comparant les valeurs de la pression, calculées numériquement à l'aide du modèle TEHD, au point $P_{2}$, on observe que :

- pour une charge imposée constante de $8 \mathrm{kN}$, la pression varie de 1,24 MPa pour une vitesse de 2600 tr.min $^{-1}$ à $1,32 \mathrm{MPa}$ pour une vitesse de

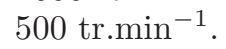


Tableau 2. Caractéristiques mécaniques de la butée.

\begin{tabular}{lccc}
\hline Grandeur & Grain fixe & Grain mobile & Unités \\
\hline Masse volumique $: \rho$ & 8940 & 7800 & $\mathrm{~kg} \cdot \mathrm{m}^{-3}$ \\
Module d'Young $: E$ & 120000 & 210000 & $\mathrm{MPa}$ \\
Coefficient de Poisson $: \nu$ & 0,33 & 0,3 & - \\
Conductivité thermique $: K$ & 65 & 50 & $\mathrm{~W} \cdot \mathrm{m}^{-1} \cdot \mathrm{K}^{-1}$ \\
Coefficient global d'échange thermique $: h_{\text {huile }}$ & 100 & 100 & $\mathrm{~W} \cdot \mathrm{m}^{-2} \cdot \mathrm{K}^{-1}$ \\
\hline
\end{tabular}

Tableau 3. Conditions de fonctionnement.

\begin{tabular}{lcccc}
\hline Vitesse de rotation & $N$ & $500 \ldots 2600$ & tr.min $^{-1}$ \\
\hline Charge axiale appliquée & $W$ & $1000 \ldots 25000$ & $\mathrm{~N}$ \\
\hline Température d'alimentation & $T_{0}$ & 45 & ${ }^{\circ} \mathrm{C}$ \\
\hline
\end{tabular}

Tableau 4. Caractéristiques du lubrifiant.

\begin{tabular}{lccc}
\hline Lubrifiant & - & ISO VG 32 & - \\
\hline Viscosité dynamique à $40{ }^{\circ} \mathrm{C}$ & $\mu_{40}$ & 0,0293 & Pa.s \\
\hline Viscosité dynamique à $70{ }^{\circ} \mathrm{C}$ & $\mu_{70}$ & 0,0111 & $\mathrm{~Pa} . \mathrm{s}$ \\
\hline Conductivité thermique & $\mathrm{K}_{\mathrm{h}}$ & 0,13 & $\mathrm{~W} \cdot \mathrm{m}^{-1} \cdot \mathrm{K}^{-1}$ \\
\hline Masse volumique & $\rho$ & 870 & $\mathrm{~kg} . \mathrm{m}^{-3}$ \\
\hline
\end{tabular}

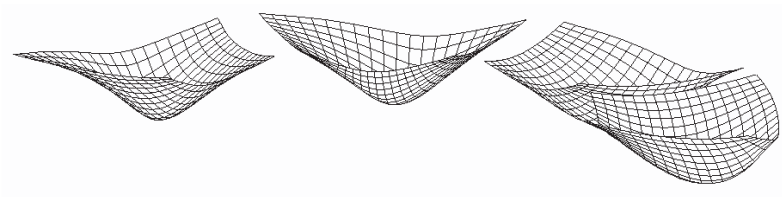

Fig. 5. Déplacements de la surface des 4 patins.

- pour une vitesse de rotation constante, fixée à 2600 tr. $\mathrm{min}^{-1}$, la pression maximale passe de 0,13 $\mathrm{MPa}$ pour une charge de $1 \mathrm{kN}$ à $1,23 \mathrm{MPa}$ pour une charge de $8 \mathrm{kN}$ et elle atteint 4,07 MPa en appliquant une charge de $25 \mathrm{kN}$.

La pression hydrodynamique varie fortement avec la charge appliquée (Figs. 11a et d) car l'intégration du champ de pression sur tout le contact lubrifié doit équilibrer l'effort extérieur. Le champ de pression, et en particulier la pression au centre du patin, sont peu sensibles à la variation de la vitesse de rotation du collet, lorsque la charge appliquée reste constante. Cependant, on observe une répartition de pression qui évolue légèrement avec la vitesse car le champ de température et la géométrie du film changent avec les conditions de fonctionnement.

\subsubsection{Influence des déformations mécaniques sur l'épaisseur minimale du film d'huile}

Les comparaisons de l'épaisseur minimale du film mesurée par Dadouche [6], et celle obtenue numériquement avec ou sans la prise en compte des déformations mécaniques, dans des diverses conditions de fonctionnement, sont présentées sur les figures 12 et 13. L'épaisseur

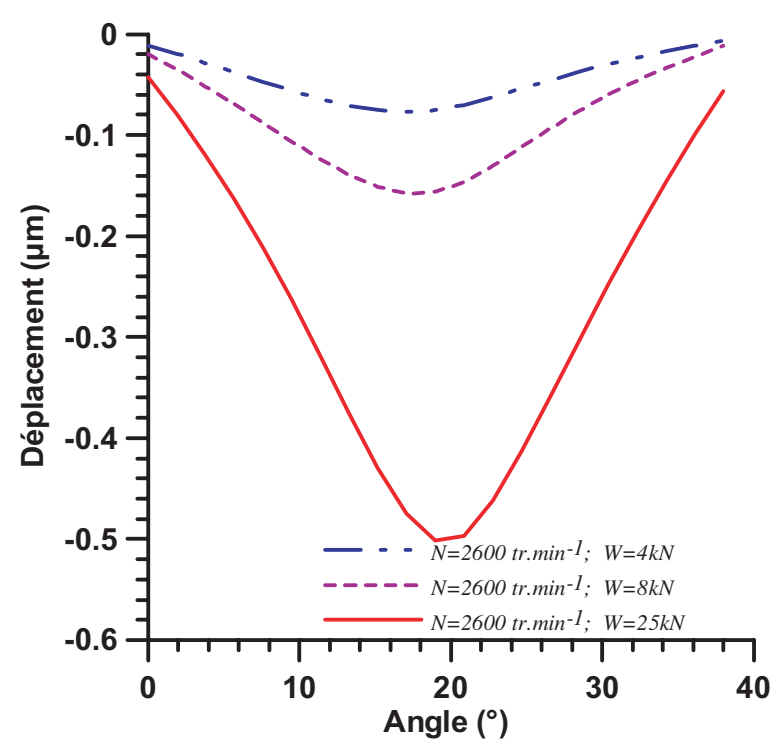

Fig. 6. Déplacements axiaux au rayon moyen du patin $(R=$ $76,50 \mathrm{~mm}$ ) suivant la direction angulaire.

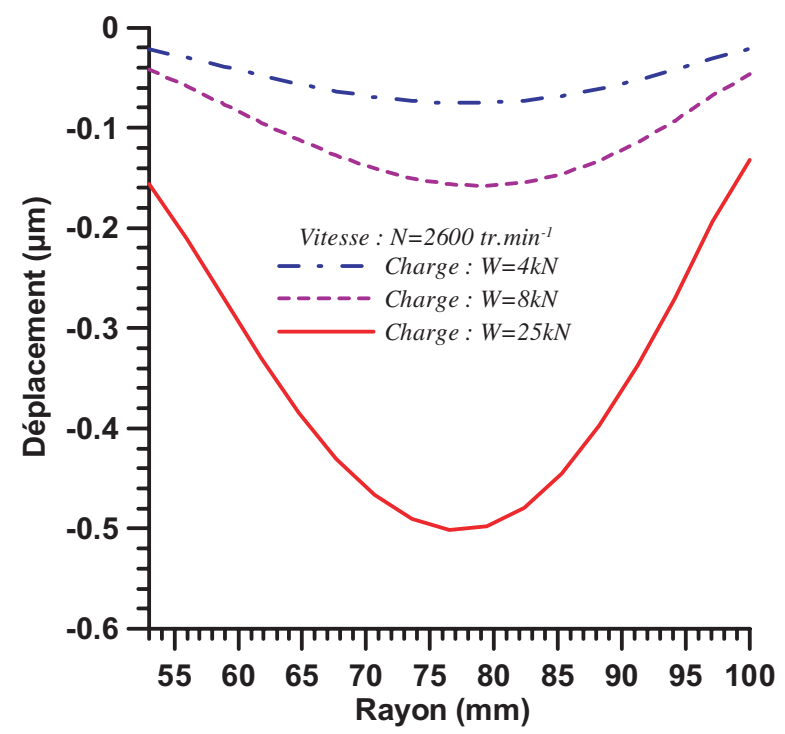

Fig. 7. Déplacements axiaux à mi-amplitude angulaire du patin $\left(\theta=19^{\circ}\right)$ suivant la direction radiale.

minimale du film augmente rapidement avec les faibles vitesses de rotation. Elle croît légèrement avec les vitesses de rotation élevées (Fig. 12). En effet, les grandes vitesses engendrent des températures plus élevées et donc une viscosité du fluide dans le film lubrifiant plus faible 




Fig. 8. Déplacements axiaux de la surface d'une section du collet suivant la direction radiale.



Fig. 9. Variation de la pression $P_{2}$ avec la charge appliquée pour une vitesse de rotation constante.

qui diminue l'effet hydrodynamique dû à l'accroissement de la vitesse de rotation du grain mobile. En négligeant les effets des déformations mécaniques, nous constatons que les épaisseurs minimales du film prédites sont supérieures à celles obtenues expérimentalement ainsi qu'à celles obtenues en TEHD, mais cependant l'allure générale des courbes théoriques est respectée.

Pour une charge constante de $8 \mathrm{kN}$, l'épaisseur minimale de film en fonction de la vitesse de rotation (Fig. 12), présente des écarts entre les expériences et le modèle TEHD allant de $2,5 \%$ à $10 \%$. Les résultats numériques THD et TEHD ont des écarts allant de $8 \%$ à $18 \%$ (entre

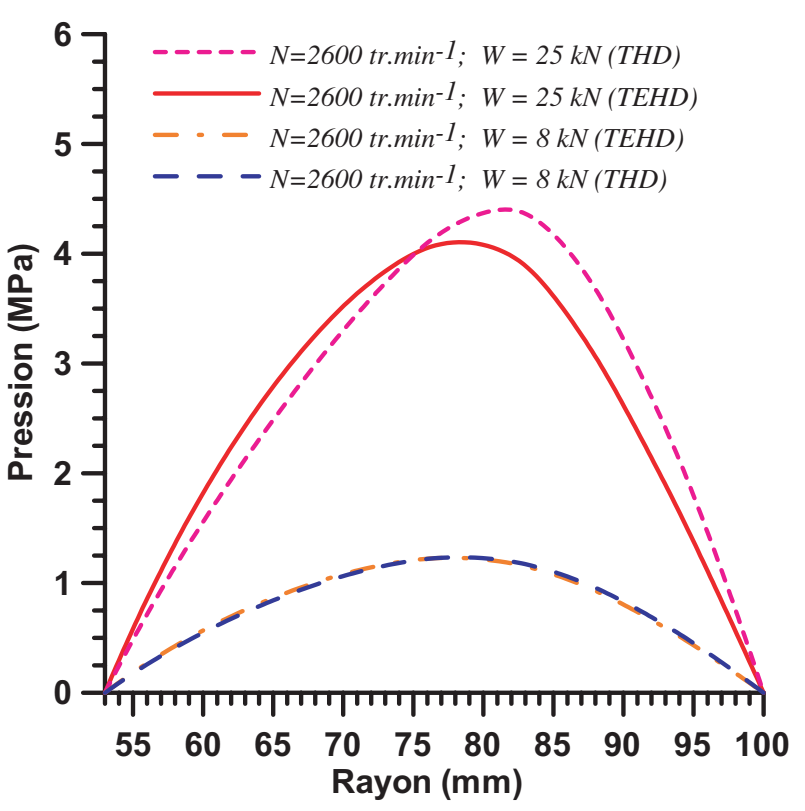

Fig. 10. Répartition radiale de la pression à mi-amplitude angulaire du patin $\left(\theta=19^{\circ}\right)$ suivant la direction radiale.

les expériences et le modèle THD, les écarts vont de $15 \%$ à $20 \%$ ).

L'épaisseur minimale du film décroît avec la charge appliquée, et de façon significative lorsque celle-ci est faible (Fig. 13).

De même pour une vitesse de rotation fixée à 2600 tr.min ${ }^{-1}$ (Fig. 13), l'épaisseur minimale de film en fonction de la charge appliquée présente des écarts sensibles entre la théorie THD et la théorie TEHD aux fortes charges appliquées. La tendance de la théorie TEHD est particulièrement bonne en se référant à celle donnée par les expériences réalisées pour une charge inférieure ou égale à $8 \mathrm{kN}$. Les écarts observés entre les résultats des deux modèles numériques vont de $0,2 \%$ à $22 \%$.

Pour les charges inférieures ou égales à $8 \mathrm{kN}$, les résultats issus du modèle TEHD sont proches des données expérimentales; les écarts varient entre $8 \%$ et $22 \%$. Les approches THD et TEHD ont des écarts significatifs dès que les charges sont élevées, la diminution de l'épaisseur minimale du film se fait progressivement en fonction de la charge appliquée (Fig. 13).

Pour une vitesse de rotation élevée constante de 2600 tr. $\mathrm{min}^{-1}$, l'évolution de la répartition de l'épaisseur du film, suivant la direction radiale, calculée sur la surface du patin à mi-amplitude angulaire est donnée par les courbes de la figure 14. Les écarts sont également significatifs dès que les conditions de fonctionnement deviennent de plus en plus sévères.

On ne peut donc pas négliger les déformations mécaniques pour déterminer avec précision l'épaisseur minimale du film des butées hydrodynamiques à géométrie fixe fortement chargées. 


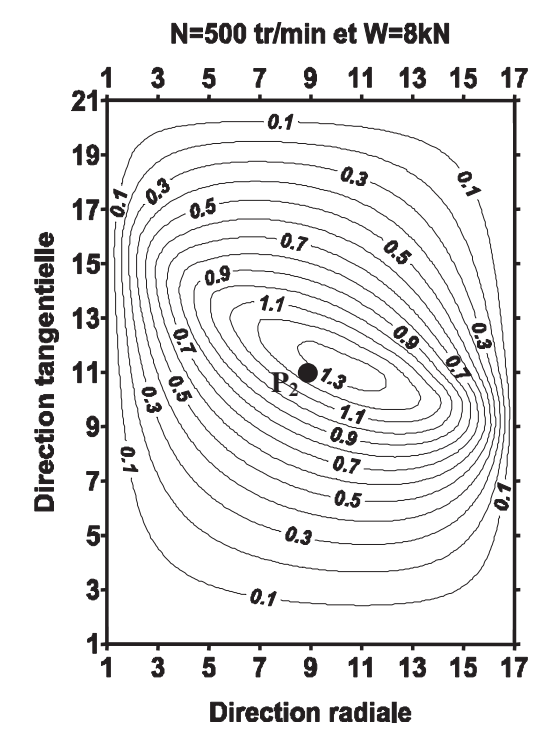

$P_{2}=1,277 \mathrm{MPa} \quad($ a)



(c)

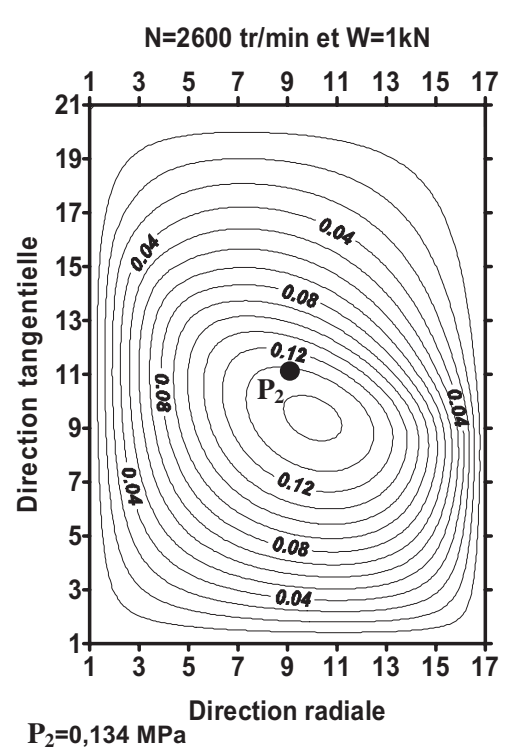

(b)



(d)

Fig. 11. Champs de pression hydrodynamique pour différentes conditions de fonctionnement, avec prise en compte des déformations mécaniques.

\subsubsection{Influence des déformations mécaniques sur le champ de température}

L'évolution de la température à la surface active d'un patin a été relevée par Dadouche [6], à l'aide d'un ensemble de dix thermocouples de type $\mathrm{K}$ (ChromelAlumel) : neuf sont implantés dans l'un des patins et le dixième est implanté à l'entrée de la rainure d'alimentation. La température maximale est relevée dans la zone de sortie au rayon extérieur, le thermocouple correspondant est défini par le point de coordonnées polaires :

$T_{9}\left(r=92,5 \mathrm{~mm}, \theta=35^{\circ} \mathrm{C}\right)$ suivant un repère lié au patin (Fig. 1). Les courbes des figures 15 et 16 correspondent aux températures mesurées et calculées au point $T_{9}$.
Dans le cas d'une charge constante de $8 \mathrm{kN}$ (Fig. 15), l'augmentation de la vitesse de rotation a pour conséquence une augmentation plus ou moins sensible de la température de la butée et globalement la théorie TEHD concorde avec les expériences réalisées ainsi qu'avec la théorie THD.

La figure 16 présente la variation de la température maximale en fonction de la charge appliquée pour une vitesse de rotation constante égale à $2600 \mathrm{tr}$. $\mathrm{min}^{-1}$. Aux faibles charges, la théorie THD est presque confondue avec la théorie TEHD avec un très léger rapprochement de la théorie THD aux expériences réalisées. La concordance la plus significative de la théorie TEHD avec les expériences est constatée aux plus grandes charges. Ceci peut s'expliquer par le fait qu'à faible charge, la 


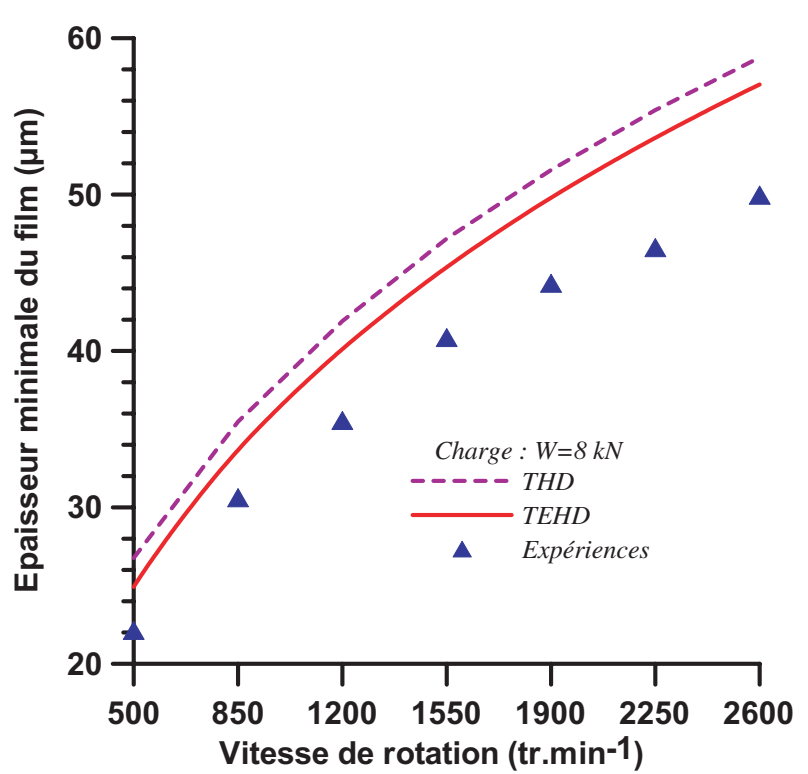

Fig. 12. Épaisseur minimale du film en fonction de la vitesse de rotation pour une charge constante.

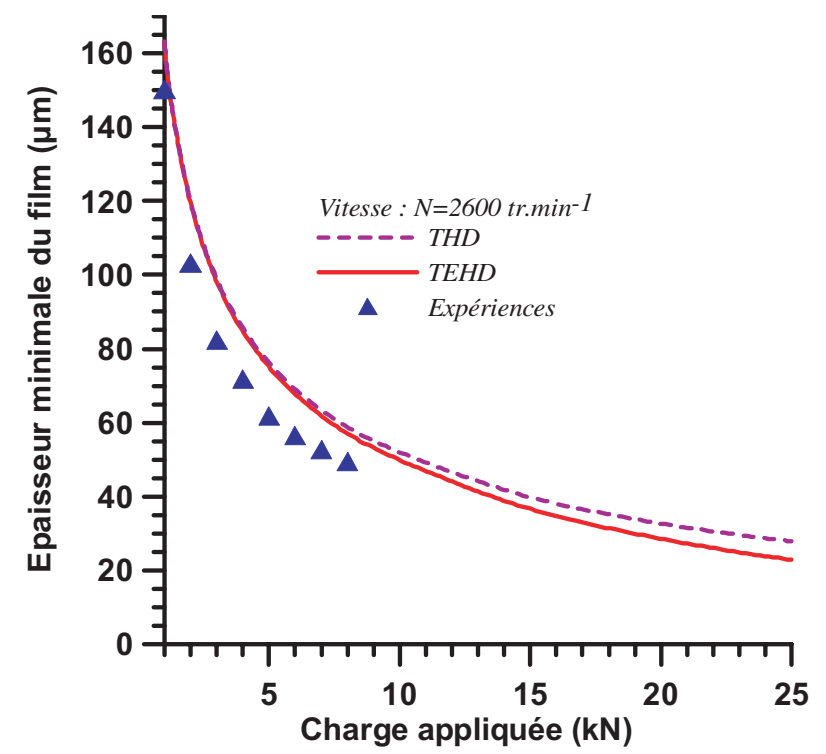

Fig. 13. Épaisseur minimale du film en fonction de la charge appliquée pour une vitesse de rotation constante.

température maximale est peu affectée par la vitesse de rotation, car le fluide est peu cisaillé.

C'est dans les conditions extrêmes de fonctionnement que nous avons utilisées $\left(N=2600\right.$ tr.min ${ }^{-1}$ et $W=$ $25 \mathrm{kN}$ ) que la température maximale est atteinte, c'est une conséquence directe de la forte sollicitation du fluide : dans ce cas, la vitesse tangentielle est élevée et l'épaisseur minimale du film très faible (Fig. 14).

Les figures 17 à 20 montrent les variations radiales et circonférentielles de la température à l'interface film/patin au niveau des rayons et des angles où sont placés les thermocouples. Nous nous intéressons, particulièrement, aux rayons intérieur et extérieur ainsi qu'à l'entrée et à la sortie des patins. L'ensemble de ces

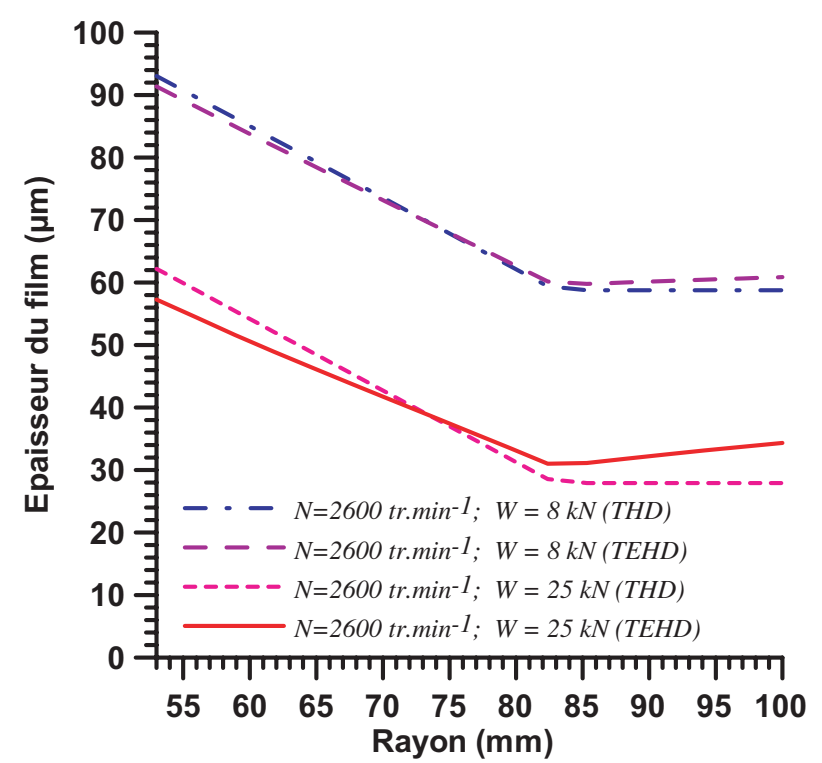

Fig. 14. Variation de l'épaisseur du film calculée dans la section centrale du patin suivant la direction radiale pour un angle fixé à $19^{\circ}$.

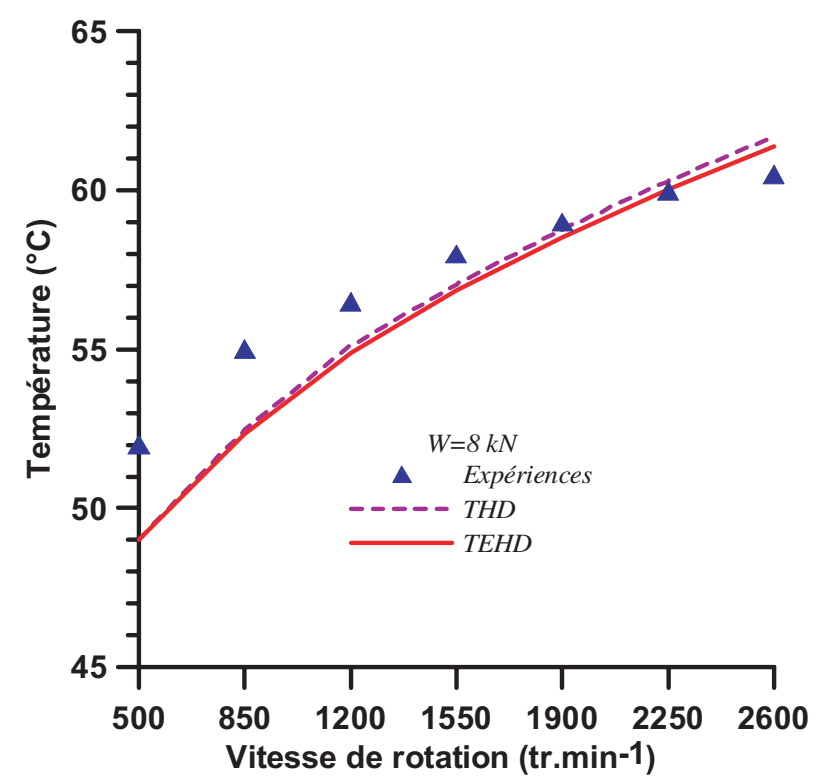

Fig. 15. Variation de la température maximale (T9) en fonction de la vitesse de rotation pour une charge constante.

graphes concerne les conditions extrêmes de fonctionnement que nous avons utilisées $\left(N=2600 \mathrm{tr}^{-\mathrm{min}^{-1}}\right.$ et $W=25 \mathrm{kN}$ ). Ces courbes mettent en évidence les écarts observés entre la théorie THD et la théorie TEHD qui sont significatifs aux fortes sollicitations. Les effets des déformations mécaniques deviennent non négligeables si l'on veut prédire correctement le champ de température et en particulier la température maximale. Il y a peu de variation circonférentielle de la température au rayon $R=62,5 \mathrm{~mm}$ (Fig. 17) alors qu'elle devient importante au rayon $R=92,5 \mathrm{~mm}$ (Fig. 18). Il y a également très peu de variation radiale de la température à l'entrée du 


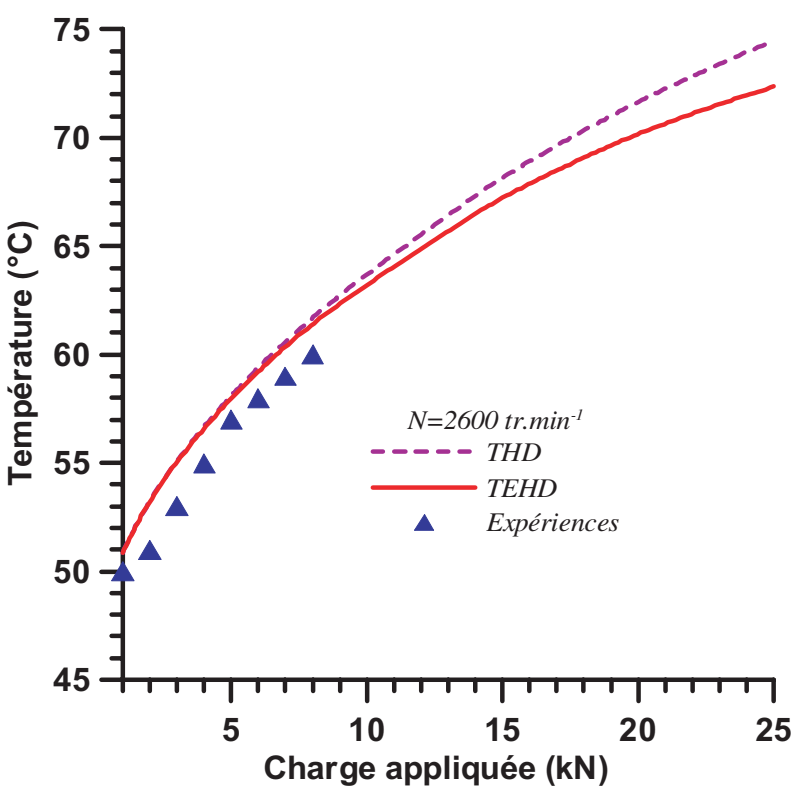

Fig. 16. Variation de la température maximale (T9) en fonction de la charge appliquée pour une vitesse de rotation constante.

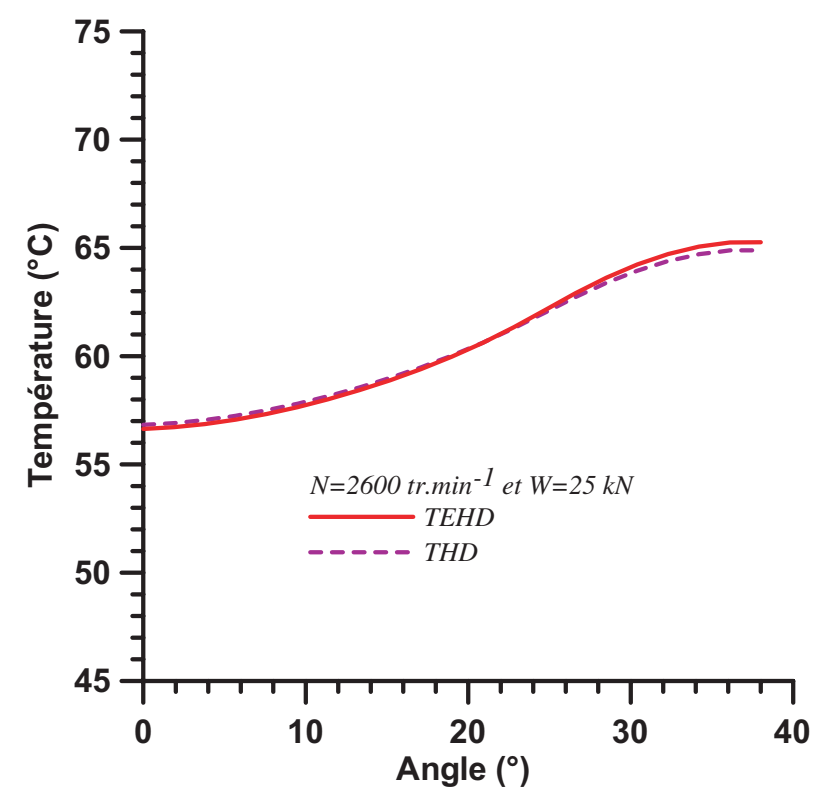

Fig. 17. Variation de la température à l'interface film/patin dans la direction angulaire pour un rayon fixé à $62,50 \mathrm{~mm}$ à charge et vitesse constantes.

patin (Fig. 19) alors qu'elle est significative à la sortie du patin (Fig. 20).

\subsubsection{Comparaison des résultats (expériences, THD et TEHD)}

Par rapport au modèle THD, les calculs qui tiennent compte des déformations mécaniques diminuent sensiblement l'épaisseur minimale du film, pour les charges

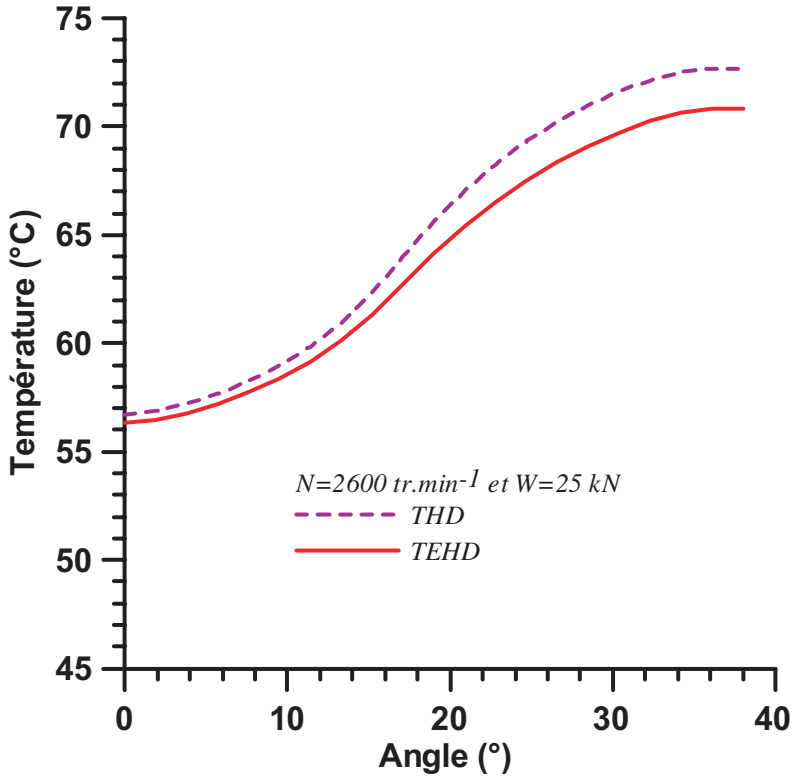

Fig. 18. Variation de la température à l'interface film/patin dans la direction angulaire pour un rayon fixé à $92,50 \mathrm{~mm}$ à charge et vitesse constantes.

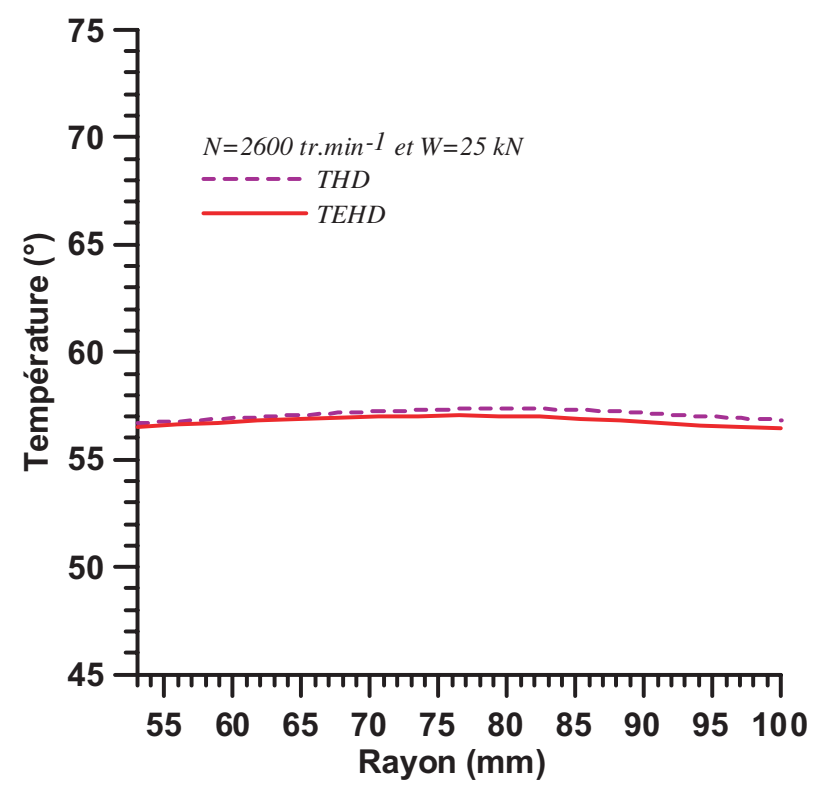

Fig. 19. Variation de la température à l'interface film/patin dans la direction radiale à l'entrée du patin $\left(\theta=3^{\circ}\right)$ à charge et vitesse constantes.

élevées, et modifient les autres caractéristiques de fonctionnement qui deviennent également plus proches des résultats expérimentaux. Les paramètres les plus affectés par les déplacements du grain fixe et du grain mobile sont la température et l'épaisseur du film d'huile. On constate une diminution de la température maximale (Fig. 16) ainsi qu'une diminution de la température à la sortie du patin (Fig. 20) et au rayon extérieur (Fig. 18).

Cette étude met en évidence l'importance des déformations mécaniques des éléments de la butée pour 


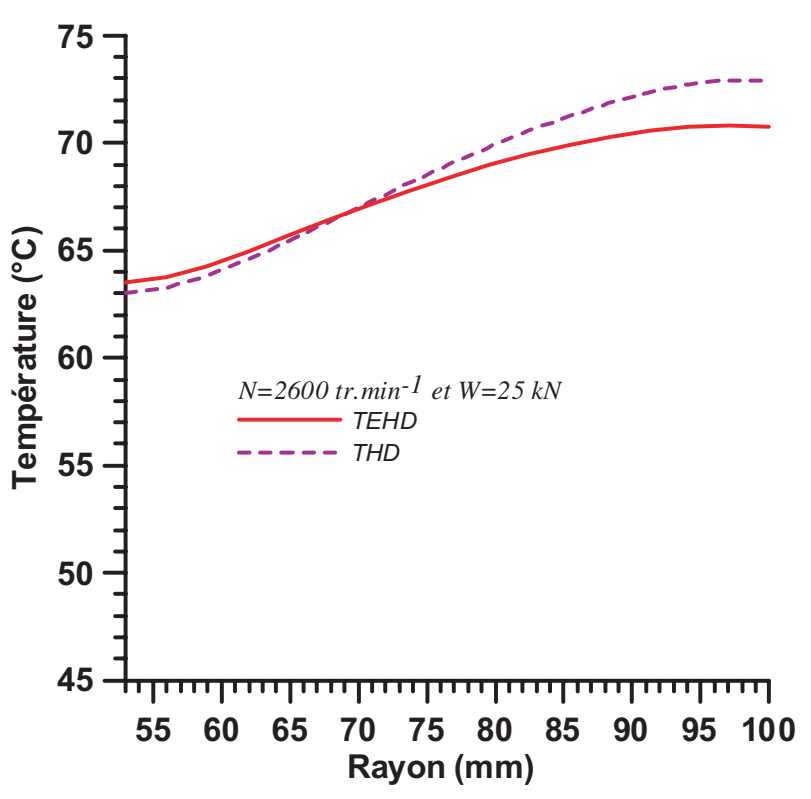

Fig. 20. Variation de la température à l'interface film/patin dans la direction radiale à la sortie du patin $\left(\theta=35^{\circ}\right)$ à charge et vitesse constantes.

la détermination de ses performances telles la pression et la température maximale ainsi que l'épaisseur minimale du film lubrifiant. Pour des butées faiblement chargées (charges inférieures ou égales à $4 \mathrm{kN}$ ), les déformations du patin sont quasi négligeables et n'ont pas de conséquence sur les performances de la butée. Pour les fortes charges, les déformations de la butée et en particulier celles du collet ont un rôle très important sur les caractéristiques de fonctionnement de la butée.

\section{Conclusion}

Les phénomènes thermiques et le rôle des déformations mécaniques dans une butée hydrodynamique à géométrie fixe ont été étudiés. La confrontation entre les résultats issus des simulations numériques et ceux obtenus à partir des expérimentations est particulièrement bonne dès qu'on prend en compte les déformations mécaniques. Les déformations mécaniques augmentent avec la charge appliquée, ce qui diminue progressivement l'épaisseur minimale du film lubrifiant et pourra, dans les cas extrêmes, ainsi causer la destruction du système.

Il ressort que pour déterminer précisément les caractéristiques de fonctionnement d'une butée hydrodynamique à patins fixes, il ne faut pas négliger les déformations mécaniques du collet, en particulier, lorsque la charge appliquée est élevée. Nous préconisons donc l'utilisation de collets plus résistants aux déformations, son épaisseur étant dans ce contexte un facteur important, sujet à optimisation. Le collet doit être suffisamment épais pour résister aux déformations mécaniques mais suffisamment mince pour ne pas avoir des butées massives.

Les petites différences observées lors de la comparaison des résultats numériques aux résultats expérimentaux peuvent être dues par la non prise en compte, dans le modèle TEHD, des déformations thermiques des éléments de la butée. Il conviendra donc d'en tenir compte dans nos prochains travaux.

\section{Références}

[1] O. Pinkus, Anisothermal Fluid film in Tribology, Isr. J. Tribology 22 (1984) 120-141

[2] M.M. Khonsari, A Review of Thermal Effects in Hydrodynamic Bearing Part 2: J. bearings, ASLE Transactions (1987) 26-34

[3] M. Fillon, J. Frêne, R. Boncompain, Historical aspect and present development on thermal effects in hydrodynamic bearings, Proc. of 13th Leeds-Lyon Symposium on tribology, 1987, pp. 27-47

[4] M. Tanaka, Recent Thermohydrodynamic Analysis and Designs of Thick-Film Bearing, Proc. IME, J. Eng. Tribology 214 (2000) 107-121

[5] A. Dadouche, M. Fillon, Analyse théorique et expérimentale des effets thermiques dans les butées hydrodynamiques à géométrie, Mécanique \& Industries 1 (2000) 141-150

[6] A. Dadouche, M. Fillon, J.C. Bligoud, Experiments on thermal effects in a hydrodynamic thrust bearing, Tribology Int. (2000) 167-174

[7] C.M.M. Ettles, Transient Thermoelastic Effects in Fluid Film Bearings, Wear 79 (1982) 53-71

[8] C.M.M. Ettles, S. Adavni, The control of thermal and elastic effects in thrust bearings, Proc. of the 6th Leeds Lyon Symposium, 1980, pp. 105-116

[9] S. Brockett, L. Barrett, P.E. Allaire, Thermoelastohydrodynamic analysis of fixed geometry thrust bearing including runner deformation, Tribology Transactions 39 (1996) 555-562

[10] K.H. Huebner, Solution for the Pressure and Temperature in Thrust Bearing operating in the Thermohydrodynamic Turbulent Regime, ASME, J. Lubrication Technology 96 (1974) 58-68

[11] K.H. Huebner, A Three Dimensionnal Thermohydrodynamic Analysis of sector Thrust Bearings, ASLE Transactions 17 (1974) 62-73

[12] A. Dadouche, M. Fillon, W. Dmochowski, Performance of hydrodynamic fixed geometry thrust bearing: comparison between experimental data and numerical results, Tribology Transactions 49 (2006) 419-426

[13] R.D. Richtmyer, Difference methods for initial value problems, Interscience Publishers Inc., New York, 1957 